09,03

\title{
Невзаимные оптические и магнитооптические эффекты в полупроводниковых квантовых ямах
}

\author{
(c) Л.В. Котова ${ }^{1,2}$, А.В. Платонов ${ }^{3}$, В.Н. Кац ${ }^{3}$, Т.С. Шамирзаев ${ }^{2}$, R. André $^{4}$, В.П. Кочерешко \\ ${ }^{1}$ Университет ИТМО, \\ Санкт-Петербург, Россия \\ ${ }^{2}$ Институт фризики полупроводников им. А.В. Ржанова СО РАН, \\ Новосибирск, Россия \\ ${ }^{3}$ Физико-технический институт им. А.Ф. Иофрфе РАН, \\ Санкт-Петербург, Россия \\ ${ }^{4}$ Institute Neel, \\ Grenoble, France \\ E-mail: kotova@mail.ioffe.ru
}

В работе исследованы эффекты, проявляющиеся в нарушении законов отражения света Френеля в полупроводниковых структурах с квантовыми ямами. Это нарушение связано с проявлением пространственной дисперсии, вызванной спин орбитальным взаимодействием в структурах, не имеющих центра инверсии. Измерены величины констант спинорбитального взаимодействия, характеризующие преобразование поляризаций в симметричных и асимметричных структурах с квантовыми ямами.

Работа Котовой Л.В. и Шамирзаева Т.С. выполнена в рамках проекта РФФИ 17-32-50161.

DOI: $10.21883 /$ FTT.2018.11.46668.28NN

\section{1. Введение}

Невзаимные оптические и магнитооптические явления все больше и больше привлекают интерес исследователей в последние годы. Это связано с тем, что представляется чрезвычайно заманчивым научиться использовать невзаимные эффекты в полупроводниковой микро- и оптоэлектронике, аналогично тому как они уже используются в СВЧ радиотехнике. Полупроводниковые наноструктуры с квантовыми ямами представляются особенно перспективными для проявления невзаимных эффектов.

Замечательной особенностью невзаимных явлений является то, что скорости распространения волн в прямом и обратном направлении разные. Одно из таких явлений - явление естественной оптической активности (гиротропии). Иногда его также называют эффектом невзаимного циркулярного двулучепреломления. Не все кристаллы являются гиротропными, необходимым условием для наблюдения гиротропии является отсутствие центра инверсии в кристалле. Однако это условие не является достаточным, например, кубические кристаллы с симметрией $T_{d}[1]$ не являются гиротропными, несмотря на отсутствие центра инверсии.

Удивительно, что в неоднородной негиротропной среде гиротропия может неожиданно проявиться. Простейшим примером неоднородной среды является полубесконечная среда, например гетеропереход. Уже в полубесконечной среде с симметрией $C_{\infty v}$, составленной из изотропных сред, возможно проявление невзаимных оптических эффектов. Причина этого состоит в том, что полубесконечная среда не имеет центра инверсии. Появление невзаимных эффектов в оптически однородной, изотропной, полубесконечной среде аналогично появлению линейного по волновому вектору расщепления зоны проводимости вблизи гетероперехода, предсказанному Рашбой [2]. Эффект Рашбы для электронов подробно изучался в полупроводниках. Однако для света подобные эффекты до сих пор не наблюдались.

Для классификации различных оптических явлений удобно использовать разложение тензора диэлектрической восприимчивости по степеням волнового вектора света и внешних электрического и магнитного полей

$$
\chi_{i j}(\mathbf{B}, \mathbf{q})=\chi_{i j}^{0}+S_{i j k} B_{k}+i \gamma_{i j k} q_{k}+C_{i j k} B_{k} q_{1}+\ldots
$$

Здесь волновой вектор q целиком лежит в плоскости квантовой ямы, первое слагаемое описывает частотную дисперсию и явление двулучепреломления, следующее слагаемое, содержащее тензор третьего ранга $\hat{S}$, описывает эффект Фарадея и/или магнитооптический эффект Керра. Член, содержащий тензор $\hat{\gamma}$, описывает явление гиротропии, последнее слагаемое в (1), билинейное по магнитному полю $\mathbf{B}$ и волновому вектору $\mathbf{q}$ описывает эффект магнитоиндуцированной пространственной дисперсии $[3,4]$. Как известно, в квантовых ямах восприимчивость $\chi\left(z, z^{\prime}, \mathbf{q}_{\|}\right)$зависит от $z$ и $z^{\prime}$, а не от их разности, т.е. восприимчивость становится нелокальной и может быть представлена интегральным оператором [5]. Тем не менее, разложение ядра этого оператора по степеням волнового вектора, лежащего в плоскости ямы, остается справедливым. При этом коэффициенты разложения (1) остаются нелокальными по $z$.

В кристаллах невзаимные оптические эффекты обычно связаны с наличием нечетных по волновому вектору слагаемых в тензоре диэлектрической восприимчивости. 
Их появление может быть вызвано как асимметрией кристаллической структуры (асимметрия элементарной ячейки), так и асимметрией структуры как целого. В первом случае линейный по волновому вектору вклад в диэлектрический тензор связывают с именем Г. Дрессельхауса [6], во втором случае - с именем Э.И. Рашбы. Для электронов влияние этих асимметрий на спиновую релаксацию широко исследовалось [7]. В настоящей работе ставится задача исследовать проявление этих вкладов в оптике экситонов.

\section{2. Теория}

Рассмотрим структуры с различной симметрией при отсутствии и наличии магнитного поля определенной ориентации и выпишем для каждого случая вклад в восприимчивость, зависящий от волнового вектора в плоскости ямы и магнитного поля.

\section{1. Квантовые ямы с симметрией $D_{2 d}$ без магнитного поля}

Рассмотрим тензор восприимчивости $\chi_{i j}(\omega, \mathbf{q})$ для квантовой ямы с симметрией $D_{2 d}$ с учетом только линейной пространственной дисперсии. Воспользовавшись методом инвариантов $[8,9]$, можно определить линейно независимые компоненты тензоров, входящих в формулу (1). В симметричных квантовых ямах вклад в линейную пространственную дисперсию вносит только механизм Дрессельхауса.

$$
\chi_{i j}(\omega, \mathbf{q})=\left(\begin{array}{ccc}
\chi_{\perp}^{0}(\omega) & 0 & i \gamma(\omega) q_{y} \\
0 & \chi_{\perp}^{0}(\omega) & i \gamma(\omega) q_{x} \\
-i \gamma(\omega) q_{y} & -i \gamma(\omega) q_{x} & \chi_{\|}^{0}(\omega)
\end{array}\right) .
$$

Здесь оси $x, y$ направлены вдоль кристаллографических осей [100], [010]. Ось $z$ направлена вдоль оси КЯ.

Из вида этого тензора следует, что благодаря эффектам пространственной дисперсии происходит смешивание продольной компоненты поляризации и поперечной компоненты. Если на образец падает электромагнитная волна с волновым вектором $\mathbf{q}=\left(0, q_{y}, q_{x}\right)$ под углом падения $\theta$, линейно поляризованная в поляризации (вдоль оси $x$ ), то она вызывает внутри КЯ волну, поляризованную эллиптически в плоскости $(x, z)$. Таким образом, отношение осей эллипса поляризации внутри ямы будет [10]

$$
\sim \frac{\kappa_{0}|q| \sin \theta_{i}}{\left(\omega_{\|}-\omega-i \Gamma\right)}
$$

Здесь считаем, что $\Gamma \gg \kappa_{0} q, \theta_{i}-$ угол между осью и направлением волнового вектора внутри КЯ, $\sin \theta / \sin \theta_{i}=n, n-$ показатель преломления света в образце, $\kappa_{0}$ - коэффициент при линейном по волновому вектору слагаемом в дисперсии экситона.

Отсюда получаем, что отраженный свет будет поляризованным эллиптически, а амплитуда „неправильной ${ }^{6}$
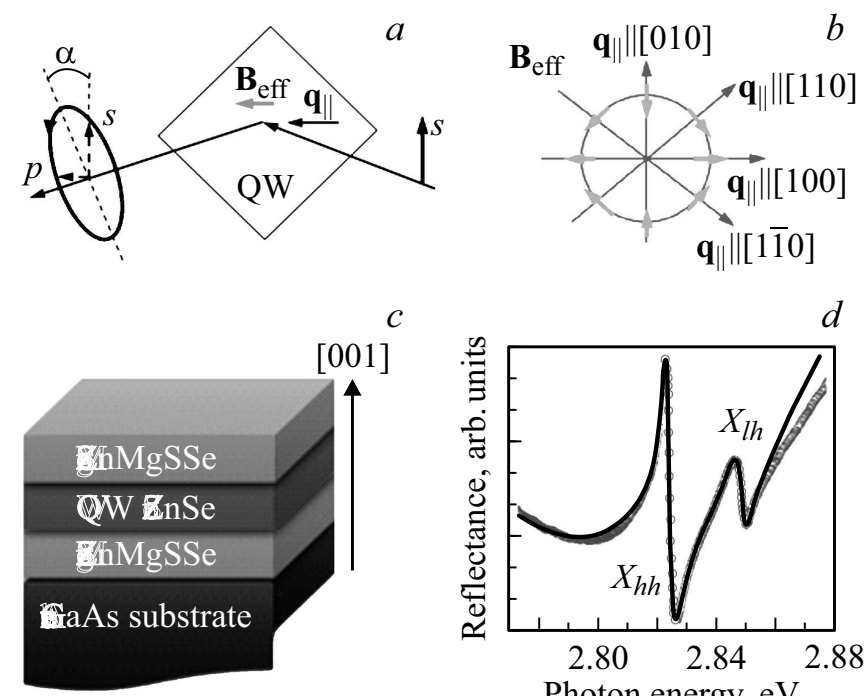

$c$

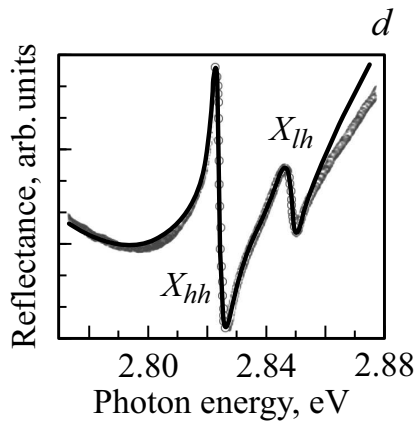

Рис. 1. a) Схема эксперимента. На образец падает свет в $s$-поляризации, анализируется поляризация отраженного света. $\mathbf{B}_{e f f}$ - эффективное магнитное поле, связанное со спин-орбитальным взаимодействием; $b$ ) Направление $\mathbf{B}_{\text {eff }}$ относительно кристаллографических осей в квантовой яме; $c$ ) Схема исследованной структуры; $d$ ) Спектр отражения от данной структуры, снятый при нормальном падении света.

поляризации, пропорциональная недиагональной компоненте коэффициента отражения $r_{s p}=r_{p s}$, будет

$$
\sim \frac{\kappa_{0}|q| \sin \theta_{i}}{\left(\omega_{\perp}-\omega-i \Gamma\right)\left(\omega_{\|}-\omega-i \Gamma\right)} \frac{\sin \theta_{i}}{\cos \theta_{i}} .
$$

Очевидно, вблизи экситонных резонансов это отношение будет максимальным. Особенно это касается области вблизи экситона с легкой дыркой, так как вблизи резонансной частоты экситона с тяжелой дыркой $\chi_{\|}(\omega)$ не имеет полюсов.

Проявление эффектов гиротропии в кристаллах эквивалентно проявлению некоторого эффективного магнитного поля $\mathbf{B}_{e f f}$, пропорционального волновому вектору экситона. Это эффективное поле связано со спин-орбитальным взаимодействием. В объемном кубическом кристалле симметрии $T_{d}$, не имеющем центра инверсии, компоненты этого поля выражаются через компоненты волнового вектора электрона $B_{e f f}=\xi\left[q_{i}\left(q_{i+1}^{2}-q_{i+2}^{2}\right)\right][5,7]$. В КЯ из-за множителей оно оказывается пропорциональным энергии размерного квантования. Это поле влияет на поляризацию отраженного света, подобного реальному магнитному полю в магнитооптическом эффекте Керра.

Это эффективное магнитное поле приводит к множеству эффектов в физике экситонов [5,11-14]. Оптическая активность вызвана той частью $\mathbf{B}_{e f f}$, которая имеет ненулевую проекцию на направление волнового вектора, рис. $1, a$. Поэтому асимметрия структуры, приводящая к $\mathbf{B}_{e f f} \perp \mathbf{q}[5]$, не проявляется в оптической активности. 
Направление $\mathbf{B}_{\text {eff }}$ зависит от ориентации волнового вектора фотона относительно кристаллографических осей, рис. $1, b$. Максимальное значение поляризационного преобразования достигается, когда плоскость падения содержит одну из кубических осей кристалла.

В осях $x^{\prime}, y^{\prime}$ направленных вдоль кристаллографиче-

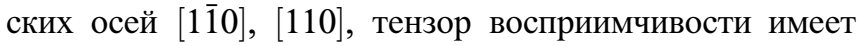
вид

$$
\chi_{i j}(\omega, \mathbf{q})=\left(\begin{array}{ccc}
\chi_{\perp}^{0}(\omega) & 0 & i \gamma(\omega) q_{x^{\prime}} \\
0 & \chi_{\perp}^{0}(\omega) & -i \gamma(\omega) q_{y^{\prime}} \\
-i \gamma(\omega) q_{x^{\prime}} & i \gamma(\omega) q_{y^{\prime}} & \chi_{\|}^{0}(\omega)
\end{array}\right) .
$$

В этом случае при отражении света с волновым вектором $\mathbf{q}=\left(0, q_{y^{\prime}}, q_{z}\right)$ отраженная волна остается линейно поляризованной.

\section{2. Квантовые ямы с симметрией $C_{\infty v}$ без магнитного поля}

В этом случае вклад в линейную пространственную дисперсию может вносить как механизм Дрессельхауса, так и механизм Рашбы.

Тензор восприимчивости $\chi_{i j}(\omega, \mathbf{q})$ для квантовой ямы с симметрией $C_{\infty v}$, записанный с учетом только линейной пространственной дисперсии

$$
\chi_{i j}(\omega, \mathbf{q})=\left(\begin{array}{ccc}
\chi_{\perp}^{0}(\omega) & 0 & i \gamma(\omega) q_{x} \\
0 & \chi_{\perp}^{0}(\omega) & i \gamma(\omega) q_{y} \\
-i \gamma(\omega) q_{x} & -i \gamma(\omega) q_{y} & \chi_{\|}^{0}(\omega)
\end{array}\right) .
$$

В этом случае также не происходит преобразования поляризаций при отражении света от структуры. Небольшая добавка линейно поляризованной компоненты в отраженный, линейно поляризованный сигнал окажется незаметной на фоне большого сигнала отражения в той же поляризации.

\section{3. КЯ с симметрией $D_{2 d}$ В магнитном поле в плоскости КЯ}

\section{1. Поле сонаправлено с волновым вектором q || B}

В данной геометрии $B=\left(B_{x}, 0,0\right), \mathbf{q}=\left(q_{x}, 0,0\right)$ тензор восприимчивости с учетом билинейного вклада по q и В имеет вид

$$
\begin{aligned}
& \chi_{i j}(\omega, \mathbf{q}, \mathbf{B})= \\
& =\left(\begin{array}{ccc}
\chi_{\perp}^{0}(\omega)+A(\omega) q_{x} B_{x} & 0 & 0 \\
0 & \chi_{\perp}^{0}(\omega) & i \gamma(\omega) q_{x}+C(\omega) B_{x} \\
0 & -i \gamma(\omega) q_{x}+C(\omega) B_{x} & \chi_{\|}^{0}(\omega)
\end{array}\right) .
\end{aligned}
$$

На легком экситоне в дополнение к гиротропии, связанной со слагаемыми $i \gamma(\omega) q_{x}$, будет наблюдаться Фарадеевское вращение. Для тяжелого экситона, линейного по магнитному полю и не зависящему от волнового вектора, вклада нет.

\section{2. Поле перпендикулярно волновому вектору $\mathbf{q} \perp \mathbf{B}$}

В этом случае $\mathbf{q}=\left(q_{x}, 0,0\right), \mathbf{B}=\left(0, B_{y}, 0\right)$ и восприимчивость может быть записана как

$$
\begin{aligned}
& \chi_{i j}(\omega, \mathbf{q})= \\
& =\left(\begin{array}{ccc}
\chi_{\perp}^{0}(\omega)+A(\omega) q_{x} B_{y} & 0 & C(\omega) B_{y} \\
0 & \chi_{\perp}^{0}(\omega)+A(\omega) q_{x} B_{y} & i \gamma(\omega) q_{x} \\
C(\omega) B_{y} & -i \gamma(\omega) q_{x} & \chi_{\|}^{0}(\omega)
\end{array}\right) .
\end{aligned}
$$

В этой геометрии возможна конверсия поляризаций. При наклонном падении света в направлении $\mathbf{q}=\left(q_{x}, 0, q_{z}\right), \quad$ с поляризацией вдоль оси $y$ $\left(s\right.$-поляризация), благодаря слагаемым $C(\omega) B_{y} \quad$ и $i \gamma(\omega) q_{x}$ на легком экситоне возможно появление компоненты, поляризованной по кругу. Если расщепление уровней легкой и тяжелой дырки в КЯ достаточно велико, то на тяжелом экситоне таких эффектов не будет. Наблюдаемый в магнитном поле эффект будет дополнительным и аналогичным по проявлению со случаем наклонного падения света в отсутствие магнитного поля. При этом он будет зависеть линейно от магнитного поля.

\section{4. КЯ с симметрией $C_{\infty v}$ в магнитном поле в плоскости КЯ}

\section{1. Поле сонаправлено с волновым вектором q || B}

Восприимчивость будет иметь вид

$$
\begin{aligned}
& \chi_{i j}(\omega, \mathbf{q}, \mathbf{B})= \\
& =\left(\begin{array}{ccc}
\chi_{\perp}^{0}(\omega)+C(\omega) q_{x} B_{x} & A(\omega) q_{x} B_{x} & i \gamma(\omega) q_{x} \\
A(\omega) q_{x} B_{x} & \chi_{\perp}^{0}(\omega)+C(\omega) q_{x} B_{x} & -\beta(\omega) B_{x} \\
-i \gamma(\omega) q_{x} & -\beta(\omega) B_{x} & \chi_{\|}^{0}(\omega)
\end{array}\right) .
\end{aligned}
$$

В данной геометрии, если предположить, что расщепление легких и тяжелых дырок достаточно велико по сравнению с зеемановским и линейным по q расщеплением, то основной эффект в преобразование поляризаций, линейной в круговую, на тяжелом экситоне будут вносить слагаемые $A(\omega) q_{x} B_{x}$. Для отношения осей эллипса поляризации тяжелого экситона будем иметь

$$
\propto \frac{a|q| B \sin \theta_{i}}{\omega_{\perp}-\omega-i \Gamma} .
$$

На легком экситоне преобразование линейной поляризации в круговую будет происходить за счет комбинированного действия $\gamma(\omega) q_{x}$ и $\beta(\omega) B_{x}$. 


\section{2. Поле перпендикулярно волновому} вектору $\mathbf{q} \perp$ B

Для восприимчивости будем иметь

$$
\begin{aligned}
& \chi_{i j}(\omega, \mathbf{q}, \mathbf{B})= \\
& =\left(\begin{array}{cccc}
\chi_{\perp}^{0}(\omega)+\tilde{A}(\omega) q_{x} B_{y} & 0 & i \gamma(\omega) q_{x}+\beta(\omega) B_{y} \\
0 & \chi_{\perp}^{0}(\omega)-\tilde{A}(\omega) q_{x} B_{y} & 0 \\
-i \gamma(\omega) q_{x}+\beta(\omega) B_{y} & 0 & \chi_{\|}^{0}(\omega)
\end{array}\right) .
\end{aligned}
$$

В данной геометрии на тяжелом экситоне преобразования поляризаций нет. На легком экситоне эффект Фарадея накладывается на эффект гиротропии, однако в спектре отражения круговая поляризация наблюдаться не будет, так как поляризация лежит в плоскости $(x, z)$.

Отметим еще раз, что для расчета коэффициентов при $q_{i}, B_{i}$ и $\chi_{0}$ следует учесть нелокальность восприимчивости по $z$.

\section{5. Эксперимент}

Исследовались три типа образцов с квантовыми ямами (КЯ).

1) Структура с одиночной, симметричной квантовой ямой $\mathrm{ZnSe} / \mathrm{Zn}_{0.64} \mathrm{Mg}_{0.18} \mathrm{~S}_{0.18} \mathrm{Se}$ была выращена на подложке [001] GaAs методом молекулярно-пучковой эпитаксии (МВЕ). Ширина квантовой ямы (КЯ) составляла $10 \mathrm{~nm}$, толщины барьеров $110 \mathrm{~nm}$, полная толщина структуры от поверхности до подложки составляла $230 \mathrm{~nm}$. Схема структуры приведена на рис. $1, c$.

2) Структура с асимметричной квантовой ямой $\mathrm{GaAs} / \mathrm{AlGaAs}$ треугольной формы была выращена методом MBE на полуизолирующей подложке $\mathrm{GaAs}$ в направлении [001]. Структура содержала барьер $\mathrm{Al}_{0.28} \mathrm{Ga}_{0.72} \mathrm{As}$ шириной $200 \mathrm{~nm}$, за которым следовала КЯ шириной $8 \mathrm{~nm}$. Затем другой барьер, наклонный, выращивали с концентрацией $\mathrm{Al}$, плавно увеличивающейся с $4 \%$ до $28 \%$ на слое шириной $27 \mathrm{~nm}$. Схема структуры приведена на рис. $2, a$. Конструкция этой структуры была аналогична конструкции образца, использованного в [7], где наблюдалась анизотропия спиновой релаксации электронов, вызванная конкуренцией механизмов Дрессельхауса и Рашбы.

3) Прямоугольная КЯ СdTe шириной $8 \mathrm{~nm}$ с барьерами различной высоты выращивалась на подложке $\mathrm{Cd}_{0.96} \mathrm{Zn}_{0.04} \mathrm{Te}$ в направлении [001]. Левый барьер представляет собой слой $\mathrm{Cd}_{0.9} \mathrm{Zn}_{0.1} \mathrm{Te}$ шириной $90 \mathrm{~nm}$, а правый барьер $\mathrm{Cd}_{0.4} \mathrm{Mg}_{0.6} \mathrm{Te}$ имеет ширину $90 \mathrm{~nm}$. Схема структуры приведена на рис. 2, $b$. На этих образцах были проведены две серии экспериментов. В первой серии экспериментов использовались структуры с симметричной КЯ $\mathrm{ZnSe} / \mathrm{Zn}_{0.64} \mathrm{Mg}_{0.18} \mathrm{~S}_{0.18} \mathrm{Se}$. Измерялись спектры поляризованного отражения от такой структуры при наклонном падении света в зависимости от угла падения и от ориентации плоскости падения по отношению к
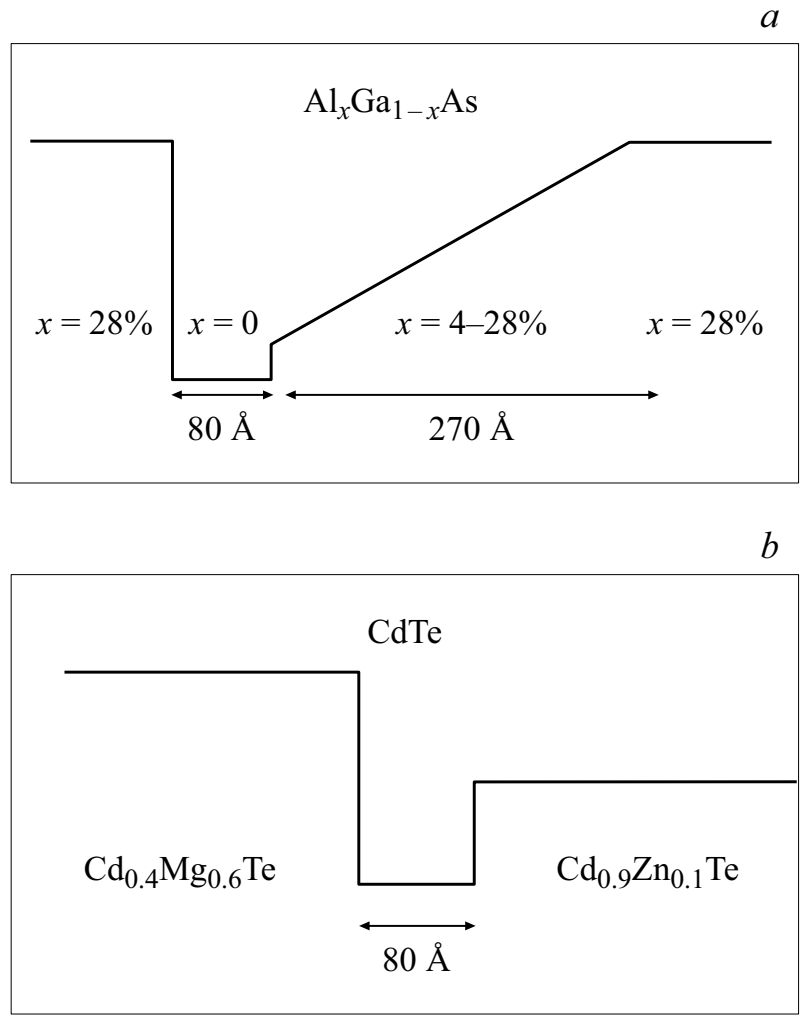

Рис. 2. Схема асимметричной структуры с квантовой ямой. a) Структура на основе GaAs с наклонными барьерами; b) структура на основе CdTe.

кристаллографическим осям образца. Схема эксперимента приведена на рис. $1, a$. Измерения проводились в стеклянном цилиндрическом криостате, который позволяет исследовать спектры отражения при произвольных углах падения. Держатель образца позволял вращать образец вокруг нормали на угол до $360^{\circ}$. Для измерения спектров отражения в качестве источника света использовалась галогенная лампа. Параллельный световой пучок был сформирован с использованием линз и щелей. Размер светового пятна превышал диаметр образца примерно в два раза.

Свет, падающий на образец, был линейно поляризован перпендикулярно плоскости падения ( $s$-поляризации). Измерялись все шесть поляризационных составляющих отраженного света, а именно интенсивности двух круговых поляризаций $I_{\sigma^{+}}, I_{\sigma^{-}}$, интенсивности двух линейных $I_{s}, I_{p}$ поляризаций, соответствующие $s$ - и $p$-поляризациям, и две линейные составляющие в осях, повернутых на $\pm 45^{\circ}$ относительно плоскости падения $I_{45}, I_{135}$. Спектры регистрировались с использованием монохроматора $0.5 \mathrm{~m}$ и CCD камеры. Мы оцениваем точность измерения степени поляризации как $0.1 \%$. Поляризация отраженного света характеризовалась параметрами Стокса

$$
P_{\text {circ }}=\frac{I_{\sigma^{+}}-I_{\sigma^{-}}}{I_{\sigma^{+}}+I_{\sigma^{-}}}, \quad P_{\text {lin }}^{45}=\frac{I_{45}-I_{135}}{I_{45}+I_{135}} .
$$


На рис. $1, d$ приведен спектр отражения, снятый от исследуемой структуры при нормальном падении света. В спектре видны две линии резонансного отражения экситонов с легкой $X_{l h}$ и тяжелой $X_{h h}$ дыркой. Спектр качественно не изменяется при изменении угла падения $\theta$. Используя расчет этого спектра, выполненный в модели нелокального отклика [5], мы определяем радиационное и диссипативное затухания легкого и тяжелого экситона. Они составляют $0.5 \mathrm{meV}$ и $35 \mathrm{meV}$ соответственно.

Было обнаружено, что вопреки законам отражения света Френеля, при падении на образец света в $s$-поляризации, в отраженном свете появляется $p$-поляризованная и циркулярно поляризованная компонента, см. pис. $1, a$. При падении на образец света в $p$-поляризации в отраженном свете появляется $s$-поляризованная, а также циркулярно поляризованная компонента. Поэтому два параметра Стокса, отсутствующих в падающей волне, $P_{\text {circ }}$ и $P_{\text {lin }}^{45}$ в отраженном свете отличны от нуля. Такая конверсия поляризаций при отражении света, падающего в $s$ или $p$ линейных поляризациях может быть обусловлена проявлением эффектов пространственной дисперсии, а именно гиротропии (рис. 3).

Максимальная величина преобразования линейной поляризации в круговую достигалась при углах падения $\theta \approx 45^{\circ}$, где оно достигает $\sim 2.5 \%$. Было установлено, что параметры Стокса отраженного света зависят от ориентации плоскости падения относительно кристаллографических осей. На рис. 4 представлена измеренная зависимость $P_{\text {circ }}(\varphi)$, где $\varphi-$ угол между плоскостью падения и осью [100]. Абсолютное значение сигнала до-

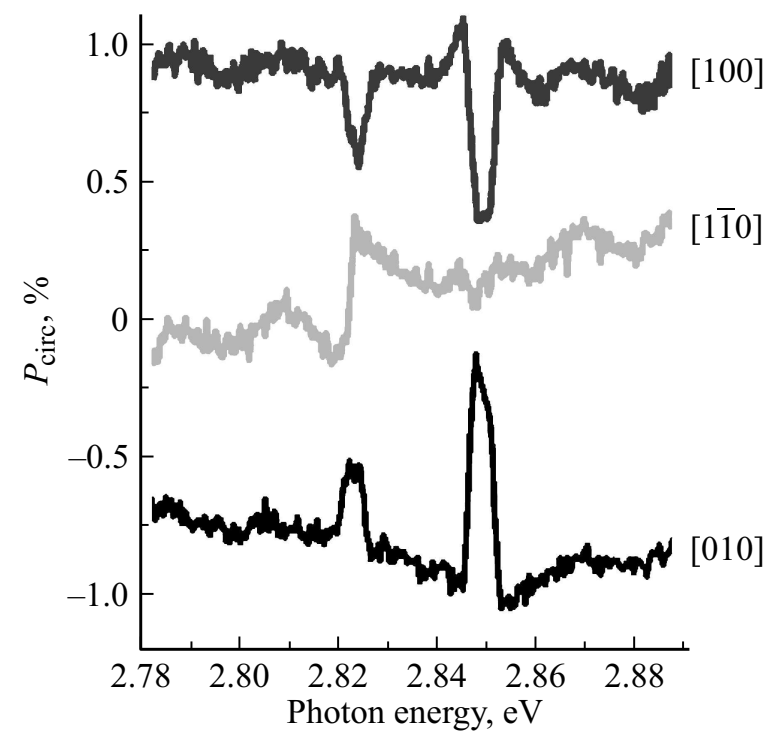

Рис. 3. Конверсия поляризаций из линейной в круговую. На образец с ZnSe KЯ во всех спектрах падал свет в линейной поляризации, перпендикулярной плоскости падения, но при разных направлениях относительно кристаллографических осей, регистрировалась круговая поляризация отраженного света. Пик на энергии $2.85 \mathrm{eV}$ относится к легкому экситону, пик на энергии $2.823 \mathrm{eV}$ относится к тяжелому экситону.

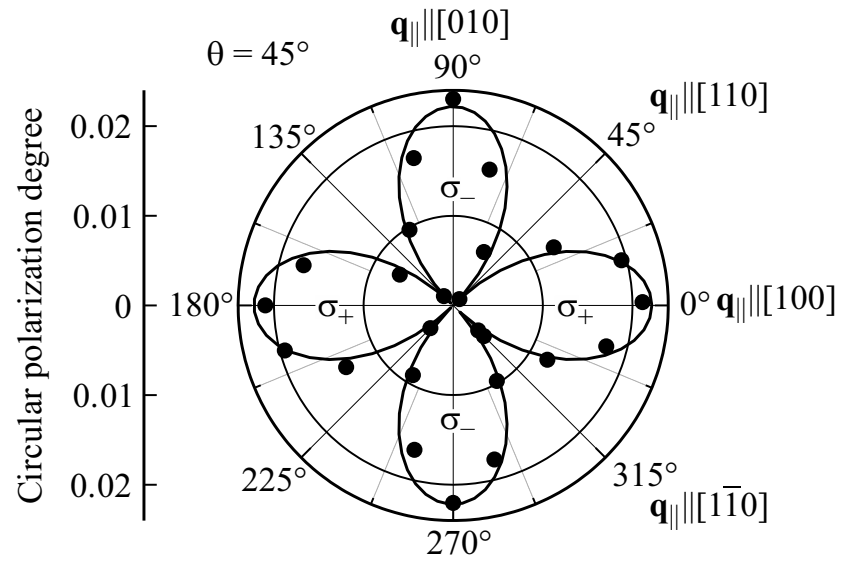

Рис. 4. Зависимость сигнала конверсии поляризаций от угла между плоскостью падения света и кристаллографическими осями для симметричной структуры с одиночной КЯ на основе $\mathrm{ZnSe}$.

стигало максимума, когда плоскость падения содержала кубические оси $[100],[010] . P_{\text {circ }}(\varphi)$ меняла знак при повороте на $90^{\circ}$ и обращалась в нуль при $q$, ориентированном вдоль направлений [110]. Такое поведение полностью отражает симметрию системы и соответствует ориентации эффективного магнитного поля $\mathbf{B}_{e f f}$, рис. $1, b$.

Во второй серии экспериментов измерялась поляризация света, отраженного от асимметричных структур с КЯ также при наклонном падении, но в присутствии магнитного поля, лежащего в плоскости падения. Падающий свет был линейно поляризован в плоскости падения ( $p$-поляризации) и/или перпендикулярно к ней $(s$-поляризацией) так же как и в предыдущей серии экспериментов. Регистрировалась интенсивность линейной и круговой поляризации отраженного света.

Геометрия эксперимента показана на рис. 5, $a$. Магнитное поле создавалось электромагнитом с ферромагнитным сердечником. Это позволило получать магнитное поле до 1 Т. Криостат с замкнутым циклом был помещен в зазор сердечника. Измерения проводились при температуре $T=3 \mathrm{~K}$. Геометрия криостата и электромагнита ограничивала максимальный угол падения света, который в нашем случае был $\theta_{0}=27^{\circ}$. Все измерения проводились именно под этим углом. Мы проверили, что при нормальном падении преобразования поляризаций при отражении света нет.

Спектр отражения образца с асимметричной КЯ на основе GaAs в окрестности экситона $X_{h h}$ с тяжелой дыркой приведен на рис. $5, b$. В спектре хорошо виден экситонный резонанс на энергии $1.551 \mathrm{eV}$. Довольно большая ширина линии $\sim 5 \mathrm{meV}$ обусловлена изменением содержания Al в градиентной яме.

Четыре поляризационные компоненты отраженного света (интенсивности в круговых поляризациях $I_{\sigma^{+}}, I_{\sigma^{-}}$ и интенсивности в линейных поляризациях под уг- 
лом $45^{\circ}$ относительно плоскости падения $\left.I_{+45}, I_{-45}\right)$ измерялись в магнитных полях от $-1 \mathrm{~T}$ до $+1 \mathrm{~T}$.

Для анализа эффектов преобразования поляризаций использовались параметры Стокса отраженного света. Параметры Стокса: степень круговой поляризации $P_{\text {circ }}=\frac{I_{\sigma^{+}}-I_{\sigma^{-}}}{I_{\sigma^{+}}+I_{\sigma^{-}}}$, степень линейной поляризации в осях $(s, p) P_{\text {lin }}=\frac{P_{s}-P_{p}}{P_{s}+P_{p}}$ и степень линейной поляризации в повернутых на $45^{\circ}$ осях $P_{\text {lin }}^{\prime}=\frac{P_{+45}-P_{-45}}{P_{+45}+P_{-45}}$.

Для того чтобы исключить влияние различных паразитных факторов, мы построили нечетный по магнитному полю вклад в степень поляризации

$$
\begin{gathered}
\rho_{\text {circ }}=\frac{P_{\text {circ }}(\mathbf{B})-P_{\text {circ }}(-\mathbf{B})}{P_{\text {circ }}(\mathbf{B})+P_{\text {circ }}(-\mathbf{B})}, \\
\rho_{\text {lin }}^{\prime}(\mathbf{B})=\frac{P_{\operatorname{lin}}^{\prime}(\mathbf{B})-P_{\operatorname{lin}}^{\prime}(-\mathbf{B})}{P_{\operatorname{lin}}^{\prime}(\mathbf{B})+P_{\operatorname{lin}}^{\prime}(-\mathbf{B})} .
\end{gathered}
$$

Эти зависимости приведены на рис. 6, когда падающий свет был в $s$ - или $p$-поляризации. С высокой точностью

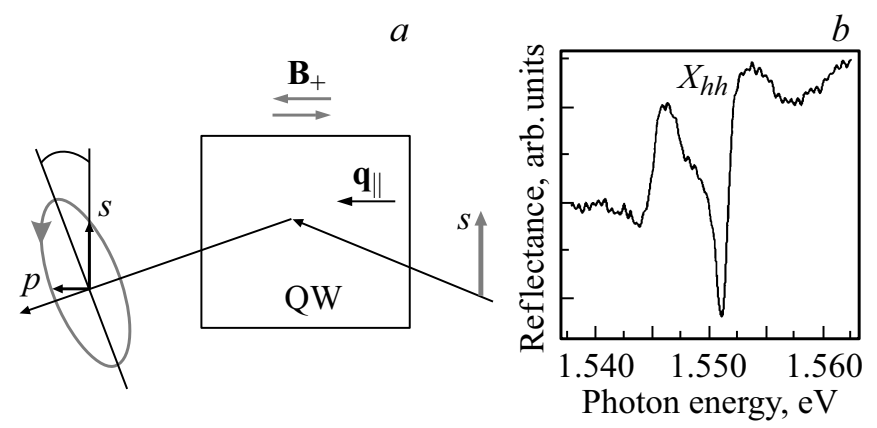

Рис. 5. a) Схема эксперимента с использованием асимметричной квантовой ямы; $b$ ) спектр отражения от структуры на основе GaAs, снятый при наклонном падении света под углом $27^{\circ}$ в $s$-поляризации. Пик на энергии $1.551 \mathrm{eV}$ соответствует тяжелому экситону.
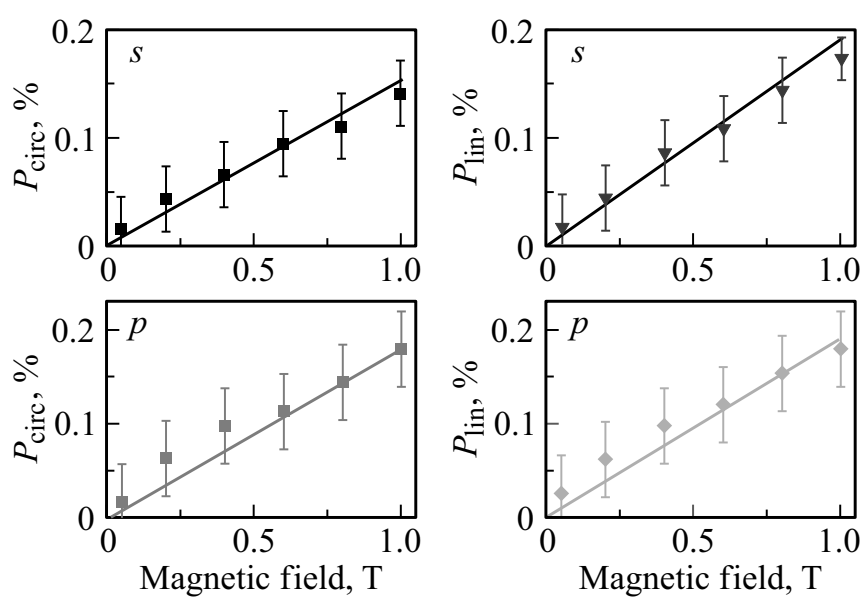

Pис. 6. Зависимости степени круговой $P_{\text {cir }}$ и линейной $P_{\text {lin }}$ поляризации отраженного света от образца асимметричной структуры на основе GaAs как функция приложенного магнитного поля, при падении света в $s$-поляризации и $p$-поляризации. все эти четыре зависимости от магнитного поля являются линейными. Видно, что зависимости для $s$ и $p$ падающих поляризаций почти одинаковы. Для структур на основе CdTe получены аналогичные зависимости, при этом величина конверсии количественно совпала с результатами для КЯ GaAs.

\section{6. Заключение}

Как известно из закона отражения света Френеля следует, что при наклонном падении на кристалл света в $s$ - или $p$-поляризации отраженный свет также должен оставаться в $s$ - или $p$-поляризациях. Однако уже давно было замечено, что это не так [15]. Причиной такого отступления от законов Френеля может являться пространственная дисперсия. В данной работе исследованы эффекты конверсии поляризаций и показано, что к эффектам преобразования поляризаций при отражении света от структур с квантовыми ямами приводят линейные по волновому вектору компоненты тензора диэлектрической восприимчивости. Влияние этих компонент многократно усиливается вблизи экситонных резонансов. Природа этих вкладов в тензор диэлектрической проницаемости связана со спин-орбитальным взаимодействием в структурах, не имеющих центра пространственной инверсии. Отсутствие центра инверсии может быть связано как с объемными свойствами кристаллической решетки, так и с асимметрией структуры в целом. В работе исследованы оба случая: конверсия поляризаций в симметричной структуре с КЯ и в асимметричной структуре в присутствии магнитного поля.

В симметричной структуре с КЯ конверсия поляризаций была обусловлена „объемным механизмом“. Из изменения амплитуды этого сигнала получена величина линейного по волновому вектору вклада в дисперсию экситона. Для симметричной КЯ на основе $\mathrm{ZnSe} / \mathrm{ZnMgSSe}$ константа спин орбитального взаимодействия (3) в экситоне $\kappa_{0} \approx 0.14 \mathrm{eV}[16]$.

Для асимметричной КЯ во внешнем магнитном поле, приложенном в плоскости падения, конверсия, зависящая от поля, может быть обусловлена только асимметрией структуры в целом. Полученные экспериментальные данные показали, что степень конверсии поляризации для структур на основе $\mathrm{CdTe}$ и $\mathrm{GaAs}$ имеет близкое значение на уровне $0.2 \%$ в поле 1 Т. Это позволило оценить константу взаимодействия, ответственную за преобразование поляризации (9) как $a \approx 0.7 \cdot 10^{-8} \mathrm{~cm} \cdot \mathrm{T}^{-1}$, где $A\left(\omega_{\perp}\right)=a q_{x} B_{x}[17]$.

\section{Список литературы}

[1] В.М. Агранович, В.Л. Гинзбург. Кристаллооптика с учетом пространственной дисперсии и теория экситонов. Наука, M. (1965). C. 374.

[2] Ю.А. Бычков, Э.И. Рашба. Письма в ЖЭТФ 39, 66-69 (1984). 
[3] Е.Л. Ивченко, В.П. Кочерешко, Г.В. Михайлов, И.Н. Уральцев. Письма в ЖЭТФ 37, 137-139 (1983).

[4] В.П. Кочерешко, В.Н. Кац, А.В. Платонов. Изв. РАН. Сер. физ. 78, 1649 (2014).

[5] E.L. Ivchenko. Optical Spectroscopy of Semiconductor Nanostructures. Alpha Science Int., Harrow, UK (2005).

[6] G. Dresselhaus. Phys. Rev. 100, 580 (1955).

[7] N.S. Averkiev, L.E. Golub, A.S. Gurevich, V.P. Evtikhiev, V.P. Kochereshko, A.V. Platonov, A.S. Shkolnik, Yu.P. Efimov. Phys. Rev. B 74, 033305 (2006).

[8] G.L. Bir, G.E. Pikus. Symmetry and strain-induced effects in semiconductors. Nauka, Moscow (1972); Wiley, N. Y. (1974).

[9] E.L. Ivchenko, G.E. Pikus. Superlattices and Other Heterostructures, Symmetry and Optical Phenomena. Springer, Berlin (1997).

[10] В.П. Кочерешко, А.Ю. Серов. Магнито-оптические явления в полупроводниках и полупроводниковых наноструктурах. Изд-во СОЛО, СПб (2017) ISBN: 978-5-98340-381-9

[11] S.D. Ganichev, L.E. Golub. Phys. Status Solidi B 251, 1801 (2014).

[12] A.V. Larionov, L.E. Golub. Phys. Rev. B 78, 033302 (2008).

[13] A.A. High, A.T. Hammack, J.R. Leonard, S. Yang, L.V. Butov, T.Ostatnick'y, M. Vladimirova, A.V. Kavokin, T.C.H. Liew, K.L. Campman, A.C. Gossard. Phys. Rev. Lett. 110, 246403 (2013).

[14] A.V. Nalitov, D.D. Solnyshkov, N.A. Gippius, G. Malpuech. Phys. Rev. B 90, 235304 (2014).

[15] Л.И. Мандельштам. Лекции по оптике, теории относительности и квантовой механике. Наука, М. (1972). С. 392.

[16] L.V. Kotova, A.V. Platonov, V.N. Kats, V.P. Kochereshko, S.V. Sorokin, S.V. Ivanov, L.E. Golub. Phys. Rev. B 94, 165309 (2016).

[17] L.V. Kotova, A.V. Platonov, V.N. Kats, V.P. Kochereshko, R. André, L.E. Golub. Phys. Rev. B 97, 125302 (2018).

Редактор Ю.Э. Китаев 